\title{
Immunization with PIII, a Fraction of Schistosoma mansoni Soluble Adult Worm Antigenic Preparation, Affects Nitric Oxide Production by Murine Spleen Cells
}

\author{
Diana Magalhães de Oliveira/*, Silvânia Antunes do Carmo, \\ David N Silva-Teixeira, Alfredo Miranda Goes/ ${ }^{+}$
}

\begin{abstract}
Departamento de Bioquímica e Imunologia, Instituto de Ciências Biológicas, Universidade Federal de Minas Gerais, Av. Antonio Carlos 6627, 30161-970 Belo Horizonte, MG, Brasil *Departamento de Medicina

Veterinária, Faculdade de Veterinária, Universidade Estadual do Ceará, Fortaleza, CE, Brasil
\end{abstract}

Nitric oxide (NO) is an important effector molecule involved in immune regulation and defense. NO produced by cytokine-activated macrophages was reported to be cytotoxic against the helminth Schistosoma mansoni. Identification and characterization of S. mansoni antigens that can provide protective immunity is crucial for understanding the complex immunoregulatory events that modulate the immune response in schistosomiasis. It is, then, essential to have available defined, purified parasite antigens. Previous work by our laboratory identified a fraction of S. mansoni soluble adult worm antigenic preparation (SWAP), named PIII, able to elicit significant in vitro cell proliferation and at the same time lower in vitro and in vivo granuloma formation when compared either to SEA (soluble egg antigen) or to SWAP. In the present work we report the effect of different in vivo trials with mice on their spleen cells ability to produce NO. We demonstrate that PIII-immunization is able to significantly increase NO production by spleen cells after in vitro stimulation with LPS. These data suggest a possible role for NO on the protective immunity induced by PIII.

Key words: Schistosoma mansoni - immunization - PIII - nitric oxide - in vitro granuloma

Nitric oxide (NO), produced at high concentrations by the inducible isoform of the enzyme NO synthase (iNOS), is an important effector molecule involved in immune regulation and defense. Besides functioning as a signaling molecule, $\mathrm{NO}$ has antimicrobial activity and is cytotoxic in processes involving increased levels of circulating bacterial toxins or cytokines (Nathan \& Hibbs 1991). In murine macrophages, a high output iNOS is regulated transcriptionally by cytokines or microbial products (Drapier 1997) and NO produced by cytokine-activated macrophages was reported to be cytotoxic against the helminth Schistosoma mansoni (James \& Glaven 1989).

$S$. mansoni has a complex life cycle in which the free-living cercaria is able to penetrate the intact skin of humans, undergo transformation into an endoparasitic larva (the schistosomulum), spend a few days in the skin, enter the venous circula-

\footnotetext{
This work received financial support from CNPq, CapesPICDT, Fapemig and PRPq-UFMG.

${ }^{+}$Corresponding author. Fax: +55-31-441.5963. E-mail: goes@mono.icb.ufmg.br

Received 4 May 1998

Accepted 31 August 1998
}

tion, migrate to the lungs and then move via the circulation to the hepatoportal circulation, where the adult worms develop, mate, and move to their final niche in the mesenteric circulation. In terms of immunity, the schistosome parasite is most susceptible to immune elimination during the skin and lung stages of development and least susceptible as an adult parasite (Capron et al. 1987, Capron 1992, Mei et al. 1996) and a possible effector molecule in such circumstances could be NO.

Previous work by our laboratory (Hirsch \& Goes 1996) identified a fraction of $S$. mansoni soluble adult worm antigenic preparation (SWAP), named PIII, that on the murine model of schistosomiasis causes protection against a challenge infection after immunization, elicits significant in vitro cell proliferation while fails to induce vigorous granulomatous hypersensitivity to eggs on liver and lungs (Hirsch et al. 1997). Recently we have described a regulatory role for $\mathrm{NO}$ on human schistosomiasis (Oliveira et al. 1998) and specially with PIII, which induces higher in vitro NO production by human mononuclear cells (Oliveira et al. unpublished observations). Attempting to further investigate whether immunization with PIII would affect the in vitro NO production, we cultured spleen cells (obtained from mice of experimental trials) with $S$. mansoni antigens and analyzed their 
nitrite releasing capacity. In the present work we report how these different treatments on groups of mice (infected, PIII-immunized or both infected and PIII-immunized) affect their spleen cells ability to produce NO.

\section{MATERIALS AND METHODS}

Parasites - S. mansoni cercariae were obtained from Biomphalaria glabrata snails previously infected with miracidia of the L.E. strain, from Belo Horizonte, Brazil. Cercariae were shed from infected snails under bright artificial illumination, as previously described (Hirsch \& Goes 1996, Hirsch et al. 1997).

Antigens for mice immunization - Antigenic preparation was obtained from SWAP, prepared as soluble supernatant fluids from buffered saline homogenates of the respective life-cycle stage (Goes et al. 1989). SWAP was fractionated by anion-exchange chromatography on FPLC (fast protein liquid chromatography), as previously described (Hirsch \& Goes 1996). Briefly, proteins were eluted with $20 \mathrm{mM}$ Tris- $\mathrm{HCl}, \mathrm{pH} 9.6$, in a multistep increasing gradient up to $1 \mathrm{M} \mathrm{NaCl}$, interrupted by hold-gradient intervals at $0,100,280$, 450, 600 and $750 \mathrm{mM}$. Flow-through fractions were concentrated by lyophilization. The concentrated material was dialyzed against $0.15 \mathrm{M}$ phosphate-buffered saline (PBS), $\mathrm{pH} 7.4$, sterilized by filtration and stored at $-70^{\circ} \mathrm{C}$. The protein content was measured according to Bradford microassay (Bradford 1976). Analysis of the six fractions, separated by $10 \%$ SDS-PAGE (sodium dodecylsulfate polyacrylamide gel electrophoresis) under reducing conditions (Laemmli 1970), showed multiple protein bands, fraction III containing high (97 and 160 $\mathrm{KDa})$, intermediate (52 and $56 \mathrm{KDa}$ ) and low (28 and $36 \mathrm{KDa}$ ) proteins. This fraction was called PIII and was used in different immunological assays.

Study population - Mice C57BL6/J (6-8 weeks old), purchased from Centro de Bioterismo, ICB, UFMG, Brazil, were maintained under standard laboratory care. Mice were divided in four main groups, each consisting of 14 mice: (i) normal control (not infected); (ii) infected with $30 \mathrm{~S}$. mansoni cercariae by anesthetically exposition on the abdomen using the ring method of Smithers and Terry (1965); (iii) immunized with $S$. mansoni antigenic fraction, PIII, by s.c. injections of $10 \mathrm{mg}$ PIII in the presence of $100 \mathrm{mg}$ of Corynebacterium parvum and $1 \mathrm{mg}$ of aluminum hydroxide $[\mathrm{Al}(\mathrm{OH}) 3]$ as adjuvant. The animals were boosted twice at 2week interval with identical amounts of antigen, but the last injection was i.p. without adjuvant; (iv) immunized with PIII, as mentioned on (iii), plus infected with 30 S. mansoni cercariae, as mentioned on (ii), thus called challenged group.
Animals were sacrificed under ether anesthesia in order to remove spleens at the 12 th week of infection/immunization.

Cell preparations - Murine spleen-derived cells, from animals of the mentioned groups, were treated with Tris-ammonium chloride, $\mathrm{pH} 7.2$, to lyse erythrocytes and then were washed with MEM. Cells were suspended with culture medium RPMI 1640 (Sigma, St. Louis, MO, USA) containing $1.6 \%$ L-glutamine, $300 \mathrm{U} / \mathrm{ml}$ of penicillin, $0.3 \mathrm{mg} /$ $\mathrm{ml}$ of streptomycin and supplemented with HEPES and $10 \%$ heat-inactivated fetal calf serum, and then counted on Neubauer chamber.

S. mansoni antigens conjugated to polyacrylamide beads $(P B)$ - $\mathrm{PB}, 40-60 \mathrm{~mm}$ in diameter (BioGel P- 40, Bio-Rad, Richmond, CA, USA), were washed in $0.5 \mathrm{M}$ bicarbonate buffer. The beads were mixed with $20 \mathrm{mg}$ of $S$. mansoni antigens obtained from adult worms (SWAP and PIII, PB-SWAP and PB-PIII), according to previously described techniques (Dougthy et al. 1984), whereas PB alone (not conjugated to any antigen) was the negative control of the reaction.

In vitro granuloma reaction - The reaction was determined utilizing antigen-coated beads, as previously described (Dougthy et al. 1984). Briefly, 200 polyacrylamide beads conjugated (or not) to S. mansoni antigens (PB, PB-SWAP and PB-PIII) were added to the bottom of 96-well tissue culture plates together with $1 \times 10^{6}$ cells/well in the presence either of $0.5 \mathrm{mM}$ of $\mathrm{Nw}$-Nitro-L-arginine methyl ester (L-NAME) from Sigma Chemical Co., (St. Louis, MO, USA), of $1 \mathrm{mg}$ of lipopolyssacharide (LPS) from Escherichia coli, (Sigma Chemical Co.), or none of them (only culture medium). The spleen cells were cultivated in a final volume of $200 \mathrm{ml}$ of RPMI with $10 \%$ heat-inactivated fetal calf serum. Each experimental and control group was set up in triplicate and maintained at $37^{\circ} \mathrm{C}$ in $5 \% \mathrm{CO}_{2}$ incubator. Cellular reactivity was determined by morphological observations, using a phase-contrast inverted tissue culture microscope (Nikon TMS, Tokyo, Japan), of visual evidence of cellular migration and adherent cell layers surrounding the beads. Granulomatous reactivity to PBSWAP and PB-PIII was compared to the nonspecific binding of mononuclear cells to polyacrylamide beads not conjugated to antigen (PB).

Nitrite determination - Nitrite concentration, an indirect measurement of NO synthesis, in supernatant culture of spleen cells on the in vitro granuloma reaction was assayed by a standard Griess reaction adapted to microplates (Green et al. 1982). Supernatants were analyzed at the 5th day of culture. The Griess reagent was prepared by mixing equal volumes of sulfanilamide $(1.5 \%$ in $5 \%$ $\left.\mathrm{H}_{3} \mathrm{PO}_{4}\right)$ and naphthylethylene diamine dihydro- 
chloride $\left(0.1 \%\right.$ in $\left.\mathrm{H}_{2} \mathrm{O}\right)$. A volume of $50 \mathrm{ml}$ of reagent was mixed with $50 \mathrm{ml}$ of supernatant and incubated at room temperature for $10 \mathrm{~min}$. Absorbance of the chromophore formed was measured at $540 \mathrm{~nm}$ using an automated microplate reader (Multiskan MCC/340, Labsystems, Finland). Nitrite concentrations were calculated by means of a $\mathrm{NaNO}_{2}$ standard curve and data were expressed as $\mathrm{mM}$ nitrite (Xiong et al. 1996).

Data analysis - Statistical analysis was assessed using the Student's t-test. A p $<0.05$ was taken as the lower limit of significance.

\section{RESULTS}

Comparative spontaneous NO production - We measured the capacity of mice spleen cells to produce $\mathrm{NO}$ using the in vitro granuloma model. Nitrite, a stable end product of NO, was investigated in culture supernatants of cells from all experimental animals at the 5th day of culture.

Cells from the infected group of mice spontaneously (in absence of LPS stimulus) produced slightly more nitrite than the control one (Fig. 1).

Cells from the PIII-immunized group did not show spontaneous release of nitrite (Fig. 2), except for a small quantity on PB treatment, demonstrating a behavior similar to control group (Fig. 1).

Challenged group (PIII-immunized and subsequently cercariae-infected) also showed diminutive spontaneous nitrite production (Fig. 3), as compared to infected group, regardless of the in vitro treatment. Marked was that PB-PIII treatment of splenocytes from challenged mice did not spontaneously release nitrite at all (Fig. 3).

Addition of the NOS competitive inhibitor, LNAME $(0.5 \mathrm{mM})$, to the culture was able to block NO production. We observed essentially equiva-

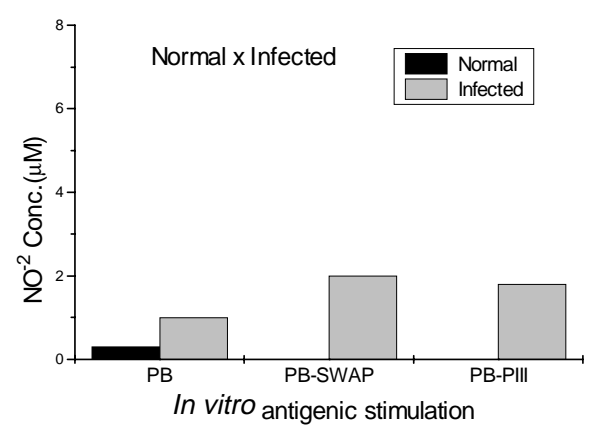

Fig. 1: comparison of spontaneous nitrite release by spleen cells on the in vitro granuloma culture. Cells from normal control $(\square)$ and infected $(\square)$ groups of mice are exposed to polyacrylamide beads (PB) coated with Schistosoma mansoni antigens (PB-SWAP and PB-PIII) or not coated (PB). Nitrite concentrations are given on $\mathrm{mM}$ and were measured at the 5th day of culture by the Griess method.

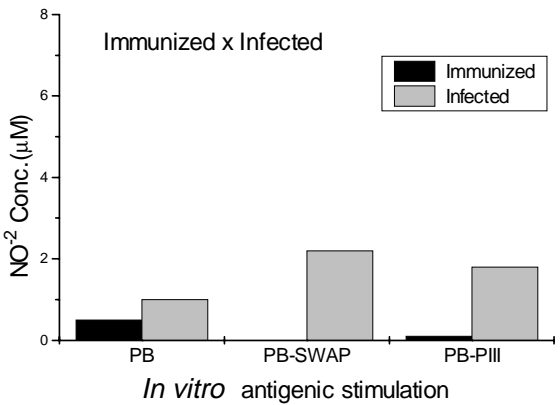

Fig. 2: comparison of spontaneous nitrite release by spleen cells on the in vitro granuloma culture. Cells from immunized ( $\square$ ) and infected $(\square)$ groups of mice are exposed to polyacrylamide beads (PB) coated with Schistosoma mansoni antigens (PBSWAP and PB-PIII) or not coated (PB). Nitrite concentrations are given on $\mathrm{mM}$ and were measured at the 5 th day of culture by the Griess method.

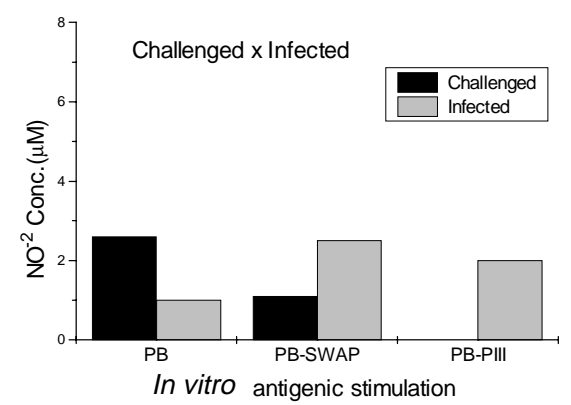

Fig. 3: comparison of spontaneous nitrite release by spleen cells on the in vitro granuloma culture. Cells from challenged ( $(\mathbf{a})$ and infected ( $\square$ ) groups of mice are exposed to polyacrylamide beads (PB) coated with Schistosoma mansoni antigens (PBSWAP and PB-PIII) or not coated (PB). Nitrite concentrations are given on $\mathrm{mM}$ and were measured at the 5 th day of culture by the Griess method.

lent degree of inhibition of nitrite production by addition of L-NAME in all compared groups (data not shown).

In vitro stimulation of splenocytes with LPS accentuates differences on nitrite production Murine spleen mononuclear cells exposed to LPS demonstrated an increasing pattern of nitrite production, usually related to the mice in vivo experimental trials, and also particularly related to the in vitro treatments (Fig. 4). PB-SWAP treatment was not able to stimulate NO production after LPS stimulation (except on the challenged group shown in Fig. 4) whereas spontaneous nitrite release did happen (Figs 1, 2, 3).

Splenocytes from not infected mice produced nitrite only after LPS-stimulation when cultivated with PB not coated to antigen. They were the only ones capable of a weak production (reaching $2 \mathrm{mM}$ 
of nitrite at the most), while splenocytes cultivated with $S$. mansoni coated antigens released no nitrite at all, even when LPS-stimulated (Fig. 4).

In the infected group, LPS did stimulate a slightly higher nitrite production only on PB treatment, but not on PB-SWAP or PB-PIII treatments (Fig. 4).

Effect of mice immunization with PIII on spleen cells ability to produce $\mathrm{NO}$ - When we measured nitrite production by spleen cells from mice of the PIII-immunization group, we observed that they characteristically released more nitrite after LPSstimulation than the control and infected groups both on PB and PB-PIII treatments. A significant difference could be seen between the immunized and the infected groups if we compare PB and PB-PIII treatments (Fig. 4). Without antigenic activation (as on PB treatment), cells from the immunized group did not show spontaneous release of nitrite, but did it when LPS-stimulated (similarly to cells from the control group), whereas on the infected group the amount of nitrite was not altered by LPS stimulation. Referring to PB-PIII treatment, there was a clear contrast in which cells from PIII-immunized mice produced nitrite (about $5 \mathrm{mM}$ ) while those from infected mice did not (Fig. 4).

Immunization with PIII and subsequent infection with cercariae (challenge) resulted in the highest NO production - Splenocytes from mice of the challenged group showed in general a higher and more constant nitrite production than the other groups for all treatments (Fig. 4). Indeed, LPS stimulation on PB-PIII treatment was responsible for the highest levels found on our experiments (around $10 \mathrm{mM}$ ).

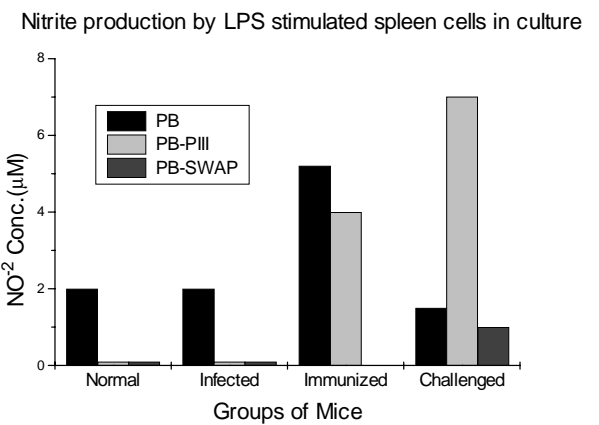

Fig. 4: LPS stimulation of spleen cells obtained from the different groups of mice and then exposed to polyacrylamide beads (PB) coated with Schistosoma mansoni antigens [PB-SWAP ( $\square$ ) and PB-PIII $\square$ ) or not coated (PB ( $\square$ ))]. Nitrite concentrations are given on $\mathrm{mM}$ and were measured at the 5th day of culture by the Griess method.

\section{DISCUSSION}

Despite its small size and transitory nature, NO is important in inflammation and immunity. Indeed, in vitro experiments demonstrated that $\mathrm{NO}$ production by cytokine-activated rodent cells is a primary mediator of their antimicrobial and antitumoral activity (Oswald \& James 1996). Abundant evidences indicate that NO contributes to the host defense functions of mononuclear phagocytes (Nathan \& Hibbs 1991, Sternberg \& McGuigan 1992, Evans et al. 1993). Referring to murine models of schistosomiasis, recently there has been a report of the increased iNOS expression in skin of mice vaccinated with cercariae of $S$. mansoni (Ramaswamy et al. 1997). However, there have been no reports of an actual nitrite in vitro production by cells that were obtained from schistosome infected mice. On the other hand nitrite production has been widely recorded in other models of diseases such as trypanosomiasis (Mabbott et al. 1994), Mycobacterium bovis (Marshall et al. 1997), Listeria monocytogenes (Xiong et al. 1996) or Dengue-virus (Mukerjee et al. 1996) infections. In the present report we have compared different in vivo experimental trials with $S$. mansoni infectedmice on their spleen cells ability to produce $\mathrm{NO}$ in vitro.

Concerning the differentially brought about splenocytes, we did observe distinguished patterns on their ability for in vitro NO production, mostly related to whether they were in vitro exposed to LPS or not. It was, then, observed that spontaneous NO production generally did not occur, no mater which in vivo trial was used to bring about splenocytes. Broadly speaking, only the in vitro LPS exposure was able to accentuate differences on NO production by splenocytes from the distinct groups of mice and also on the distinct in vitro antigenic stimuli. In fact, cells from the challenged group of mice demonstrated a comparatively remarkable NO production during in vitro $\mathrm{PB}-\mathrm{PIII}$ treatment. Data presented here suggest that NO might be involved in the immune host defense, since we observed a prominent increased NO production on PIII-immunization trials associated with the in vitro PB-PIII treatment of cells. Comparison among tested coated-antigens could distinctly confirm that total preparation of SWAP do not attend in vitro the PIII ability to activate mice spleen cells in producing NO, somewhat resembling findings we have recorded for human cells (Oliveira et al. unpublished observations). Besides that, it seems evident that, both in vivo and in vitro, PIII induces increased NO production and, since ahead of we have revealed PIII downregulating role in granuloma formation (Hirsch \& Goes 1996, Hirsch 
et al. 1997), we may speculate about a correlation between protection and NO in this case. Thus, we assumed as expected, when we found that the previously ascribed modulating features of PIII (Hirsch \& Goes 1996, Hirsch et al. 1997) extend to cause increased NO production after mice immunization with this parasite fraction. To what degree this hypothesis of NO participation may account for protection or resistance in schistosomiasis is still a speculative quantification, but some leads throw light on the question.

NO could become a critical mediator in murine schistosomiasis Th1/Th2 balance (Liew 1995). Th1 cells produce IFNg, which induces NO, whereas Th2 cells produce IL-10 and IL-4 that can inhibit iNOS at the level of transcription (Oswald et al. 1992, Liew 1995). NO is also produced by Th1 cells, whose proliferation can be inhibited by high concentrations of NO. Thus, apart from being a mediator of Th1/Th2 interaction, NO may also be an important self-regulatory molecule that prevents the over expression of Th1 cells which may be implicated in a range of severe immunopathologies (Liew 1995), including schistosomiasis (Pearce et al. 1991). A possibility that IFNginhibiting cytokines, as IL-10 and TGFb, might be involved in this process shall not be excluded, since high levels of the Th1-suppressor cytokine TGFb have been reported in schistosome infected livers of mice (Czaja et al. 1989), a condition that could be associated with the low levels of NO in schistosomiasis.

Taken together, our results suggest that NO might be responsible for some of the PIII modulating features. It is likely that NO participation on immune regulation in schistosomiasis can be greater than what has been assigned to it and, mostly, far from being elucidated. To the moment we have demonstrated that PIII treatment is able to significantly induce NO production and maybe this event has other implications on protection against $S$. mansoni infection. While activation of macrophages to kill parasites is clearly not the only mechanism capable of conferring protection against schistosome infection (Colley \& Colley 1989), the role of NO definitely requires further investigations to unveil its possible links on protection and/or pathology in schistosomiasis.

\section{REFERENCES}

Bradford MM 1976. A rapid and sensitive method for the quantification of microgram quantities of protein utilizing the principle of protein-dye binding. Analyt Biochem 72: 248-254.

Capron AR 1992. Immunity to schistosomes. Curr Opin Immunol 4: 419-424.

Capron A, Dessaint JP, Capron M, Ouma JH, Butterworth
AE 1987. Immunity to schistosomes: progress towards vaccine. Science 238:1065-1072.

Colley DG, Colley MD 1989. Protective immunity and vaccines to schistosomiasis. Parasitol Today 5: 350355.

Czaja MJ, Weiner FR, Flanders KC, Giambrone MA, Wind R, Biempica L, Zern MA 1989. In vitro and in vivo association of transforming growth factor-B1 with fibrosis. J Cell Biol 108: 2477-2481.

Dougthy BL, Ottesen EA, Nash TE, Phillips SM 1984. Delayed hypersensitivity granuloma formation around Schistosoma mansoni eggs in vitro. III. Granuloma formation and modulation in human schistosomiasis mansoni. J Immunol 133: 993-997.

Drapier JC 1997. Nitric oxide and macrophages. Pathol Biol (Paris) 45: 110-114.

Evans TG, Thai L, Granger DL, Hibbs JB 1993. Effect of in vivo inhibition of nitric oxide production in murine leishmaniasis. J Immunol 151: 907-915.

Goes AM, Rocha RS, Gazzinelli G, Doughty BL 1989. Production and characterization of human monoclonal antibodies against Schistosoma mansoni. Parasite Immunol 11: 695-711.

Green LC, Wagner DA, Glogowski J, Skipper PL, Wishnok JS, Tannenbaum SR 1982. Analysis of nitrate, nitrite and $[15 \mathrm{~N}]$ nitrate in biological fluids. Anal Biochem 126: 131-138.

Hirsch C, Goes AM 1996. Characterization of fractionated Schistosoma mansoni soluble adult worm antigens that elicit human cell proliferation and granuloma formation in vitro. Parasitology 112: 529-535.

Hirsch C, Zouain CS, Alves JB, Goes AM 1997. Induction of protective immunity and modulation of granulomatous hypersensitivity in mice using PIII an anionic fraction of Schistosoma mansoni . Parasitology 115: 21-28.

James SL, Glaven J 1989. Macrophage cytotoxicity against schistosomula of $S$. mansoni involves arginine-dependent production of reactive nitrogen intermediates. J Immunol 143: 4208-12.

Laemmli UK 1970. Cleavage of structural proteins during the assembly of the head of bacteriophage $\mathrm{T} 4$. Nature 227: 680-685.

Liew FY 1995. Nitric oxide in infections and autoimmune diseases. Ciba Found Symp 195: 234-239.

Mabbott NA, Sutherland IA, Sternberg JM 1994. Trypanosoma brucei is protected from the cytostatic effects of nitric oxide under in vivo conditions. Parasitol Res 80: 687-690.

Marshall BG, Chambers MA, Wangoo A, Shaw RJ, Young DB 1997. Production of TNF and NO by macrophages infected with live and dead Mycobacteria and their suppression by an IL-10-secreting recombinant. Infec Immun 65: 1931-1935.

Mei H, Thakur A, Schwartz J, Lo Verde PT 1996. Expression and characterization of glutathione peroxidase activity in the human blood fluke Schistosoma mansoni. Infect Immun 64: 4299-4306.

Mukerjee R, Misra A, Chaturvedi UC 1996. Dengue virus-induced cytotoxin releases nitrite by spleen cells. J Exp Pathol 77: 45-51.

Nathan CF, Hibbs JB 1991. Role of nitric oxide synthe- 
sis in macrophage antimicrobial activity. Curr Opin Immunol 3: 65-70.

Oliveira DM, Silva-Teixeira DN, Carmo SA, Goes AM 1998. Role of nitric oxide on human schistosomiasis mansoni: upregulation of in vitro granuloma formation by L-NAME. Nitric Oxide 2: 57-65.

Oswald IP, James SL 1996. Nitrogen oxide in host defense against parasites. Methods 10: 8-14.

Oswald IP, Gazzinelli RT, Sher A, James SL 1992. IL10 synergizes with IL-4 and TGFb to inhibit macrophage cytotoxic activity. J Immunol 148: 35783582.

Pearce EJ, Caspar P, Grzych JM, Lewis FA, Sher A 1991. Downregulation of Th1 cytokine production accompanies induction of $\mathrm{Th} 2$ responses by a parasitic helminth Schistosoma mansoni. J Exp Med 173: 159166.
Ramaswamy K, He YX, Salafsky B 1997. ICAM-1 and iNOS expression increased in the skin of mice after vaccination with gamma-irradiated cercariae of Schistosoma mansoni. Exp Parasitol 86: 118-132.

Smithers SR, Terry RJ 1965. The infection of laboratory hosts with cercariae of Schistosoma mansoni and the recovery of adult worms. Parasitology 55: 565-570.

Sternberg J, McGuigan F 1992. Nitric oxide mediates suppression of T-cell responses in murine Trypanosoma brucei infections. Eur J Immunol 22: 2741 2744.

Xiong H, Kawamura I, Nishibori T, Mitsuyama M 1996. Suppression of IFNg production from Listeria monocytogenes specific T-cells by endogenously produced nitric oxide. Cell Immunol 172: 118-125. 